\title{
Recycling and Reuse of Sulfur Hexafluoride in Guangdong Power Grid Corporation
}

\author{
Min Chen \\ Guangdong Power Grid Co., Ltd. Yuedian Building, Dongfengdong Street, Guangzhou, China \\ cmah05@163.com
}

\begin{abstract}
Keywords: $\mathrm{SF}_{6}$, recycling and reuse, "SF 6 Purified in Multi-channel (SFPM)" technology Abstract. As an excellent insulating medium, sulfur hexafluoride $\left(\mathrm{SF}_{6}\right)$ is widely used in electrical equipment, despite its high global warming potential. Besides, a series of hazardous decomposition products can be formed after long-term use of $\mathrm{SF}_{6}$, so it is very important for power companies to recycle $\mathrm{SF}_{6}$ in electrical equipment that is in overhaul or to be retired. To solve this problem, a "SF 6 Purified in Multi-channel (SFPM)" technology was developed, which comprises washing, adsorption, distillation, curing and exhaust gas circulation processes. The specific purification processes by which disused $\mathrm{SF}_{6}$ is purified can be customized according to its quality and the quality of $\mathrm{SF}_{6}$ after treatment meets the standards of "Industrial Sulfur Hexafluoride" (GB/T 12022-2006) and IEC 60480-2004, with zero emission of $\mathrm{SF}_{6}$ achieved during the whole process. Equipment for $\mathrm{SF}_{6}$ purification was developed and a base for $\mathrm{SF}_{6}$ recycling and reuse was established in 2010. During the past six years of operation and promotion, $93.630 \mathrm{t}$ of $\mathrm{SF}_{6} \mathrm{was}$ recycled and a total of $90.887 \mathrm{t}$ of treated $\mathrm{SF}_{6}$ was reused in electrical equipment at Guangdong Power Grid Corporation.
\end{abstract}

\section{Introduction}

Because of its unique insulating properties, high arc extinguishing properties, low toxicity and chemical stability, $\mathrm{SF}_{6}$ is widely used in electrical equipment such as Gas Insulated Switchgear (GIS), high-voltage transformers, high voltage circuit breakers and gas insulated transmission lines (GIL) ${ }^{[1]}$. According to the Intergovernmental Panel on Climate Change, $\mathrm{SF}_{6}$ is also the most potent greenhouse gas with a global warming potential of 23,900 times that of $\mathrm{CO}_{2}$ when compared over a

100-year period and a long atmospheric lifetime $(\sim 3200 \text { years })^{[2]}$. After a long-term use of $\mathrm{SF}_{6}$ in

electrical equipment, a series of hazardous decomposition byproducts can be formed due to high voltage electric field effect, electrical equipment discharge failure and other factors, such as $\mathrm{SF}_{4}$, $\mathrm{S}_{2} \mathrm{~F}_{10}, \mathrm{SOF}_{2}, \mathrm{SO}_{2} \mathrm{~F}_{2}, \mathrm{SOF}_{4}, \mathrm{~S}_{2} \mathrm{~F}_{10} \mathrm{O}, \mathrm{HF}, \mathrm{SO}_{2}$, etc ${ }^{[3]}$, which are all toxic and hazardous to human beings. If a leak or discharge may occur, workers and the people living around would pose a serious threat.

About 6,000 tons of $\mathrm{SF}_{6}$ gas is produced in China each year, more than $50 \%$ of which is used in the power industry, and the amount of $\mathrm{SF}_{6}$ to be replaced in repair process is about 700 tons ${ }^{[4]}$. $\mathrm{SF}_{6}$ gas consumption in power equipment industry is about 3,010 tons in 2008, about 4,590 tons in 2011 and about 4,890 tons in 2012, which shows a rapid growth rate. As the largest provincial power grid company in China, Guangdong Power Grid Corporation was equipped with lots of $\mathrm{SF}_{6}$ electrical equipment, the amount of $\mathrm{SF}_{6}$ in which has increased to 2300 tons and the amount of $\mathrm{SF}_{6}$ leakage has increased to about 40 tons by 2015 . It is very important for power companies to recycle $\mathrm{SF}_{6}$ in electrical equipment that is in overhaul or to be retired.

Some methods have been developed for $\mathrm{SF}_{6}$ purification, such as thermal treatment [5], membrane-based separation technique ${ }^{[6]}$, and adsorption treatment ${ }^{[7]}$, but these methods are not 
applicable to purify $\mathrm{SF}_{6}$ gas in large quantities quickly and efficiently. According to the special demand of $\mathrm{SF}_{6}$ gas treatment in Guangdong Power Grid Corporation, a "SF 6 Purified in Multi-channel (SFPM)" technology was developed, which comprises washing, adsorption, distillation, curing and exhaust gas circulation processes, which can change the purification processes and speed according to the quality of $\mathrm{SF}_{6}$ gas.

\section{Main Contents}

The biggest difficulty in $\mathrm{SF}_{6}$ gas purification is that how to detect and cleanup the impurity components, such as moisture, acidity, decomposition products and air, because these impurity components are low in mass ratio, changes in large range, and all need special treatment methods. Due to the special nature of impurity components in $\mathrm{SF}_{6}$ gas and the large amount of $\mathrm{SF}_{6}$ gas to be treated every year in Guangdong Power Grid Corporation, a new method should be developed to purify $\mathrm{SF}_{6}$ gas that is different in impurity components quickly and efficiently. It is the actual demand to recycle and reuse of $\mathrm{SF}_{6}$ in Guangdong Power Grid Corporation.

Quality standard of SF6 gas in China

The purity and components of $\mathrm{SF}_{6}$ gas are key factors for its insulation properties in electrical equipment, so the quality of $\mathrm{SF}_{6}$ gas is strictly standardized to keep the safety of power grid. In Guangdong Power Grid Corporation, "Sulfur hexafluoride for industrial use (GB/T 12022 2006)" works as $\mathrm{SF}_{6}$ gas quality standards, which proposed eight indicators, such as purity of $\mathrm{SF}_{6}$, mass fraction of air, mass fraction of $\mathrm{CF}_{4}$, humidity, mass fraction of acidity (calculated in $\mathrm{HF}$ ), hydrolysable fluorides (calculated in HF), mass fraction of mineral oil, and toxicity.

Table 1 Quality standard of SF6 gas in China

\begin{tabular}{cc}
\hline Indicators & Limits \\
\hline Purity of $\mathrm{SF}_{6}$ & $\geq 99.9 \%$ \\
Mass fraction of air & $\leq 0.04 \%$ \\
Mass fraction of $\mathrm{CF}_{4}$ & $\leq 0.04 \%$ \\
Humidity & $\leq 0.0005 \%$ \\
Mass fraction of acidity (calculated in HF) & $\leq 0.00002 \%$ \\
Hydrolysable fluorides (calculated in HF) & $\leq 0.00010 \%$ \\
Mass fraction of mineral oil & $\leq 0.0004 \%$
\end{tabular}

Toxicity

Non-toxic in biological experiments

Each indicator represents a special damage to the insulation property of $\mathrm{SF}_{6}$ gas and the electrical equipment, so the $\mathrm{SF}_{6}$ gas in any electrical equipment would be replaced to keep safe, if one or more indicator exceeds the limit in table 1.

Disused $\mathrm{SF}_{6}$ gas in Guangdong Power Grid Corporation

Disused $\mathrm{SF}_{6}$ gas was created in three ways. The first, $\mathrm{SF}_{6}$ gas of substandard quality was detected in routine test; the second, $\mathrm{SF}_{6}$ gas in faulty equipment was collected in repair process; and the last, $\mathrm{SF}_{6}$ gas in retired equipment was collected in dismantling process. Due to the large amount of $\mathrm{SF}_{6}$ gas, different equipments and variety of application environment, the disused $\mathrm{SF}_{6}$ gas in Guangdong Power Grid Corporation is really complicated from component content to component concentration.

a. $\mathrm{SF}_{6}$ gas of substandard quality

$\mathrm{SF}_{6}$ gas of substandard quality accounts for about $60 \%$ of the all disused $\mathrm{SF}_{6}$ gas in Guangdong Power Grid Corporation. The first reason of substandard quality is purity of $\mathrm{SF}_{6}$, which accounts for about $60 \%$; the second reason of substandard quality is humidity, which accounts for about $25 \%$; the third reason of substandard quality is mass fraction of air, which accounts for about $12 \%$. Component content changes with the source of the $\mathrm{SF}_{6}$ gas.

b. $\mathrm{SF}_{6}$ gas in faulty equipment

$\mathrm{SF}_{6}$ gas in faulty equipment accounts for about $35 \%$ of the all disused $\mathrm{SF}_{6}$ gas in Guangdong Power Grid Corporation. Due to some equipment accidents happen occasionally, a large amount of $\mathrm{SF}_{6}$ gas is was recovered to prevent the greenhouse effect. The $\mathrm{SF}_{6}$ gas in faulty equipment is a little special because there may be many kinds of byproducts which are difficult to be separated. 
c. $\mathrm{SF}_{6}$ gas in retired equipment

$\mathrm{SF}_{6}$ gas in retired equipment accounts for about $5 \%$ of the all disused $\mathrm{SF}_{6}$ gas in Guangdong Power Grid Corporation. Substandard quality of $\mathrm{SF}_{6}$ gas was not detected in routine test, but the component content in $\mathrm{SF}_{6}$ gas may be very complex.

The range of component concentration is analyzed in table 2, which shows the complexity of disused $\mathrm{SF}_{6}$ gas and importance of a flexible purification process.

Table 2 component concentration of disused $\mathrm{SF}_{6}$ gas

\begin{tabular}{ccc}
\hline Indicators & Range & Exceedance probability \\
\hline Purity of $\mathrm{SF}_{6}$ & $90.0 \% \sim 99.9 \%$ & $51 \%$ \\
Mass fraction of air & $0.02 \% \sim 0.80 \%$ & $20 \%$ \\
Mass fraction of $\mathrm{CF}_{4}$ & $0.01 \% \sim 0.10 \%$ & $4 \%$ \\
Humidity & $0.0002 \% \sim 0.0100 \%$ & $12 \%$ \\
Mass fraction of acidity (calculated in HF) & $0.00001 \% \sim 0.0020 \%$ & $8 \%$ \\
Hydrolysable fluorides (calculated in HF) & $0.00005 \% \sim 0.00300 \%$ & $1 \%$ \\
Mass fraction of mineral oil & $0.0002 \% \sim 0.0020 \%$ & $4 \%$ \\
\hline
\end{tabular}

$\mathrm{SF}_{6}$ Purified in Multi-channel (SFPM)

According to the component content of impurities $(0.1 \%$ to $10.0 \%)$ and chemical properties (melting point $-62{ }^{\circ} \mathrm{C}$, boiling point $-51{ }^{\circ} \mathrm{C}$ ) of $\mathrm{SF}_{6}$, a "SF 6 Purified in Multi-channel (SFPM)" technology was developed, which comprises pretreatment, washing, adsorption, distillation, curing and exhaust gas circulation processes. The specific purification processes by which disused $\mathrm{SF}_{6}$ is purified can be customized according to its quality and the quality of $\mathrm{SF}_{6}$ after treatment meets the standards of "Industrial Sulfur Hexafluoride" (GB/T 12022-2006) and IEC 60480-2004, with zero emission of $\mathrm{SF}_{6}$ achieved during the whole process.

"SF 6 Purified in Multi-channel (SFPM)" technology compose with 5 purification steps (as shown in Figure 1), changes in several ways according the quality of $\mathrm{SF}_{6}$ gas, and can give more than 5 solutions to speed up the purification process. SFPM technology purifies slightly polluted $\mathrm{SF}_{6}$ gas in short process and purifies heavily polluted $\mathrm{SF}_{6}$ gas in long process. No matter how great the variety of $\mathrm{SF}_{6}$ gas is, it would be purified in appropriate process.

Step 1: Gas Pretreatment. The gasification process of $\mathrm{SF}_{6}$ is endothermic reaction, so gas pretreatment with hot water bath is required to prevent liquid $\mathrm{SF}_{6}$ at the bottom of the cylinder curing, which would result in low recovery, low speed and gas waste. By heating the gas cylinder, the speed and recovery of $\mathrm{SF}_{6}$ gas gasification is improved.

Step 2: Alkaline Wash. The quality of pretreated gas is detected at on-line monitoring system to determine if an alkaline wash is necessary. Exceeded acid gases $\left(\mathrm{SO}_{2}, \mathrm{HF}\right)$ are removed in alkaline wash which is controlled by a PLC control system; so that the equipments and materials in follow process would not be damaged. The $\mathrm{SF}_{6}$ gas would be dried to remove water by a refrigeration dryer and then filtered by a floc filter to remove floc and solid impurities.

Step 3: Adsorption. The $\mathrm{SF}_{6}$ gas with little acid gases and $\mathrm{SF}_{6}$ gas treated by alkaline wash would be adsorbed by a series of Adsorbent to remove mineral oil, moisture, decomposition products, and toxic low fluoride. Considering the price and performance of catalysis, inexpensive and easily replaceable silica and activated alumina are placed upstream of the gas passage to remove impurities roughly, then A-type zeolite is placed in the medium to adsorb moisture specially, and KDHF-03 type zeolite is placed at last to as an advanced treatment of decomposition products and toxic low fluoride. The quality of $\mathrm{SF}_{6}$ would be judged after adsorption if subsequent treatment or re-adsorption is necessary. About $30 \%$ of disused $\mathrm{SF}_{6}$ gas can be purified to meet the gas quality requirements and would be separated directly. 


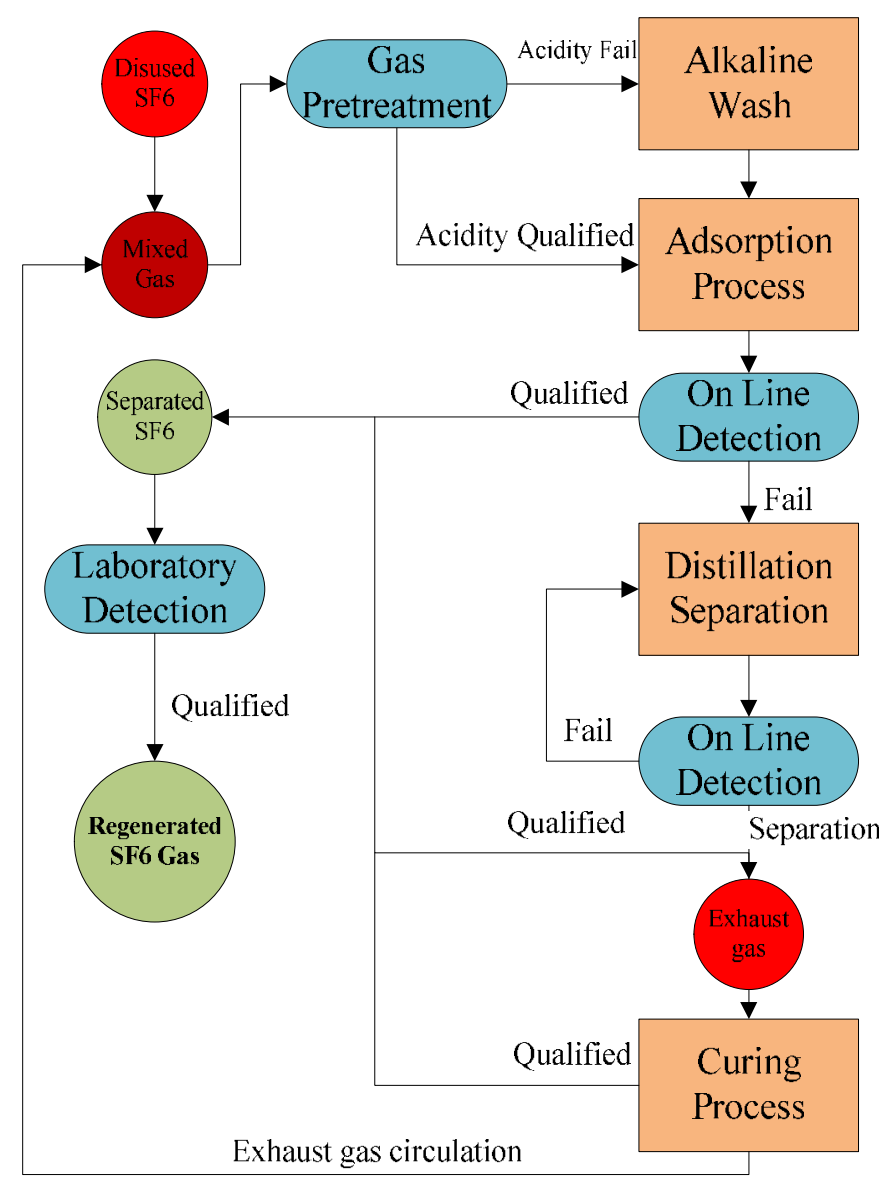

Figure. $1 \mathrm{SF}_{6}$ Purified in Multi-channel (SFPM)

Step 4: Distillation Separation. Air and remaining impurities are separated at this process quickly and efficiently. A distillation column with the processing speed of $50 \mathrm{Kg} / \mathrm{h}$ is designed under the thermodynamic theoretical calculations, which constitute the main part of SFPM technology. The unqualified $\mathrm{SF}_{6}$ gas is liquefied by a cold trap and then inputted into distillation column. The gaseous air and other remaining impurities would be separated easily because there is a great relative volatility between gaseous air and liquefied $\mathrm{SF}_{6}$. Separated $\mathrm{SF}_{6}$ is transferred to a special container, and the light component with a small amount of $\mathrm{SF}_{6}$.is transferred to an exhaust gas storage tank. If the processing efficiency is not satisfied, a re-distillation separation would be carried. About $60 \%$ of disused $\mathrm{SF}_{6}$ gas can be purified to meet the gas quality requirements at this process.

Step 5: Curing process. Zero Emission of $\mathrm{SF}_{6}$ is achieved at this step by exhaust gas treatment and recycling. The exhaust gas compressor is automatically started when the storage tank reaches a set pressure, and a cold trap under low mode would cure trace $\mathrm{SF}_{6}$.in exhaust gas. Cured $\mathrm{SF}_{6}$.is gasified and separated as clean gas. The exhaust gas would be draw out by a vacuum pump, filtered by an exhaust gas filter device, neutralized by an alkaline solution pool, mixed with untreated $\mathrm{SF}_{6}$.and retreated. About $10 \%$ of disused $\mathrm{SF}_{6}$ gas can be purified to meet the gas quality requirements at this process.

$\mathrm{SF}_{6}$ Purification Equipment

A highly integrated purification equipment(as shown in Figure 2) was develop in 2010 based on $\mathrm{SF}_{6}$ Purified in Multi-channel (SFPM) technology, which is highly automated, can be control remotely with communications technology, purify disused $\mathrm{SF}_{6}$ gas quickly and efficiently, and can regenerate gas to meet the quality standards of $\mathrm{SF}_{6}$ gas. The purification equipment is controlled by a PLC system, equipped with $\mathrm{SF}_{6}$ gas analysis instrumentation and air quality monitoring instrumentation for on line detection, and connected with chromatography instruments in laboratory by a pipe system. 
$\mathrm{SF}_{6}$ purification equipment was made in modular combinations, which means that each module is independent and can union by connecting structure. This set of equipment is divided into three major functional unit area (A, B, C zone), that are installed in guide rail and can move in direction of the specified. It is very convenient for purification equipment to be operated, maintained and repaired, because each functional area is equipped with a deployable wingspan mask.

Other characteristics of $\mathrm{SF}_{6}$ purification equipment: 1) equipped with digital weighing system, 2) zeolite being regenerated automatically, 3) equipped with remote automation operating system, 4) secondary treatment can be carried in cycle purification process, 5) zero emission of $\mathrm{SF}_{6}$ achieved.
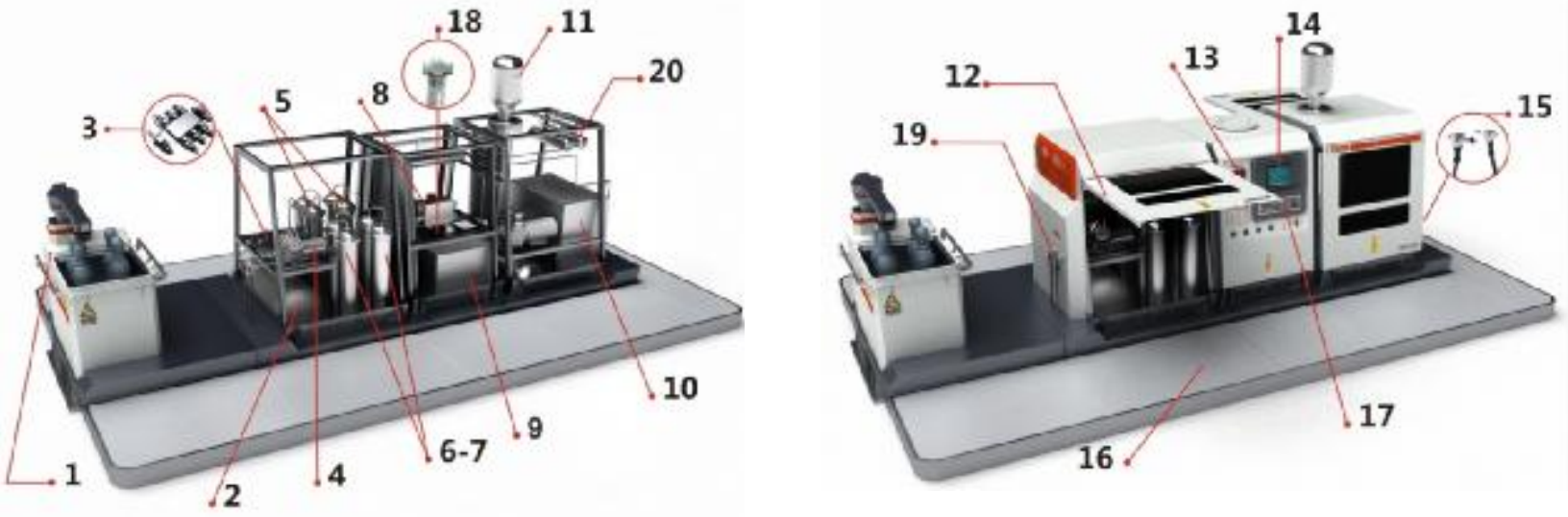

Figure. $2 \mathrm{SF}_{6}$ Purification Equipment

(1-Cylinder Pretreatment System, 2-Buffer Tank, 3-Solenoid Valve, 4-Vacuum Compressor, 5-A

Series of Zeolite, 6-7-Alkaline Wash Tank, 8-Vacuum Pump, 9-Refrigeration Dryer, 10-Cold Trap, 11-Distillation Column, 12-Wingspan Style Cover, 13-Environmental Monitoring System, 14-PLC Control System, 15-Nozzles, 16-Operator Station, 17-On Line Detecting Instrumentation, 18-SF 6 Compressor, 19-Self-styled Inlet Connector, 20-exhaust gas tank)

\section{Results}

Treatment effect of SFPM technology

In order to verify the purification efficiency of $\mathrm{SF}_{6}$ Purified in Multi-channel (SFPM) technology and $\mathrm{SF}_{6}$ purification equipment, $5 \mathrm{SF}_{6}$ gas samples was collected from 5 different transformer substations in 2010, which were named Sample A, Sample B, Sample C, Sample D and Sample E. The quality of each sample is really different and complex (as shown in Table 2), but each sample was regenerated to meet quality standard of "Sulfur hexafluoride for industrial use (GB/T 12022 2006)", which means that these regenerated $\mathrm{SF}_{6}$ could be reused to electrical equipment as insulating medium.

1) Purification efficiency of impurities

The purification efficiency of impurities by $\mathrm{SF}_{6}$ Purified in Multi-channel (SFPM) technology and $\mathrm{SF}_{6}$ purification equipment is shown in Table 2, which indicate that the 7 indicators set by "Sulfur hexafluoride for industrial use (GB/T 12022 2006)" is qualified after treatment, whatever the concentration of impurities changes drastically. 
Table 2 Purification efficiency of impurities

\begin{tabular}{ccccccccc}
\hline \multirow{2}{*}{ Samples } & Treatment & $\begin{array}{c}\mathrm{H}_{2} \mathrm{O} \\
{\left[10^{-6}\right]}\end{array}$ & $\begin{array}{c}\text { AIR } \\
{[\%]}\end{array}$ & $\mathrm{CF}_{4}[\%]$ & $\begin{array}{c}\text { Hydrolysable } \\
\text { fluorides } \\
{\left[10^{-6}\right]}\end{array}$ & $\begin{array}{c}\text { Acidity } \\
{\left[10^{-6}\right]}\end{array}$ & $\begin{array}{c}\text { Mineral } \\
\text { Oil }\left[10^{-6}\right]\end{array}$ & $\begin{array}{c}\text { Purity } \\
{[\%]}\end{array}$ \\
\hline \multirow{2}{*}{ A } & Before & 15.0 & 0.081 & 0.003 & 0.453 & 0.032 & 2.1 & 98.89 \\
& After & 2.3 & 0.010 & 0.002 & 0.032 & 0.011 & 0.2 & 99.99 \\
& Before & 13.0 & 0.006 & 0.008 & 0.684 & 0.309 & 5.7 & 99.01 \\
B & After & 1.4 & 0.005 & 0.002 & 0.083 & 0.043 & 0.3 & 99.99 \\
& Before & 12.5 & 0.072 & 0.004 & 0.365 & 0.412 & 6.1 & 99.50 \\
C & After & 1.6 & 0.008 & 0.001 & 0.032 & 0.008 & 0.5 & 99.99 \\
& Before & 10.9 & 0.056 & 0.043 & 0.451 & 0.523 & 8.2 & 98.67 \\
& After & 1.3 & 0.009 & 0.006 & 0.026 & 0.031 & 0.6 & 99.99 \\
& Before & 12.8 & 0.022 & 0.032 & 0.356 & 0.432 & 6.3 & 99.53 \\
E & After & 1.4 & 0.010 & 0.004 & 0.052 & 0.076 & 0.8 & 99.99 \\
& Standard & $\leq 5.0$ & $\leq 0.040$ & $\leq 0.040$ & $\leq 1.000$ & $\leq 0.200$ & $\leq 4.0$ & $\geq 99.9$ \\
\hline
\end{tabular}

2) Purification efficiency of subfluoride

It is difficult to remove subfluoride in $\mathrm{SF}_{6}$ gas because subfluoride is similar to $\mathrm{SF}_{6}$ in nature. As shown in Table 3, subfluoride in Sample A, Sample B and Sample C were removed thoroughly, which means that the purification efficiency of subfluoride by $\mathrm{SF}_{6}$ purification equipment is satisfied.

Table 3 Purification efficiency of subfluoride

\begin{tabular}{ccccc}
\hline Sample & Treatment & $\mathrm{SOF}_{2} / \%$ & $\mathrm{~S}_{2} \mathrm{OF}_{10} / \%$ & $\mathrm{SOF}_{4} / \%$ \\
\hline \multirow{2}{*}{$\mathrm{A}$} & Before & Undetected & 0.002 & 0.004 \\
& After & Undetected & Undetected & Undetected \\
\multirow{2}{*}{$\mathrm{B}$} & Before & 0.021 & 0.032 & Undetected \\
& After & Undetected & Undetected & Undetected \\
\multirow{2}{*}{$\mathrm{D}$} & Before & 0.011 & Undetected & 0.002 \\
& After & Undetected & Undetected & Undetected \\
\hline
\end{tabular}

3) Purification efficiency of toxicity

The purification efficiency of toxicity was tested by animal experiment. There fed mice were put in confined space that was filled with $79 \%$ regenerated $\mathrm{SF}_{6}$ and $21 \%$ oxygen. No sign of toxicity in mice was found after 48 hours, which indicate that the purification efficiency of toxicity is satisfied.

4) Insulation property of regenerated $\mathrm{SF}_{6}$

The regenerated $\mathrm{SF}_{6}$ gas was filled into a $150 \mathrm{kV}$ GIS equipment that is applied $150 \mathrm{kV}$ voltage in one electric Laboratory of Guangdong Power Grid Corporation. None partial discharge phenomenon or breakdown phenomenon was found, which indicate that the insulation property of toxicity is satisfied.

3.2 Recycling and reuse of $\mathrm{SF}_{6}$ gas in Guangdong Power Grid Corporation

A highly automated Recycling and reuse basement for $\mathrm{SF}_{6}$ gas was established in 2010 to meet the demand of $\mathrm{SF}_{6}$ gas emission reduction in Guangdong Power Grid Corporation. A life-cycle management concept of $\mathrm{SF}_{6}$ gas was carried during the process of $\mathrm{SF}_{6}$ gas emission reduction to achieve a "decentralized recycling, centralized purification and controlled reuse" model. Disused $\mathrm{SF}_{6}$ gas would be sent to this basement and reused to electric device after purification and testing qualified. 
The amount of recycled $\mathrm{SF}_{6}$ gas comes up to 93.630 tons (as shown in figure 3 ) from February 2010 to December 2015, which is equivalent to reduce 2.23 million tons of carbon dioxide emissions, and the amount of reused $\mathrm{SF}_{6}$ gas comes up to 90.887 tons, which reduced the gas purchase cost by 12.72 million Yuan.

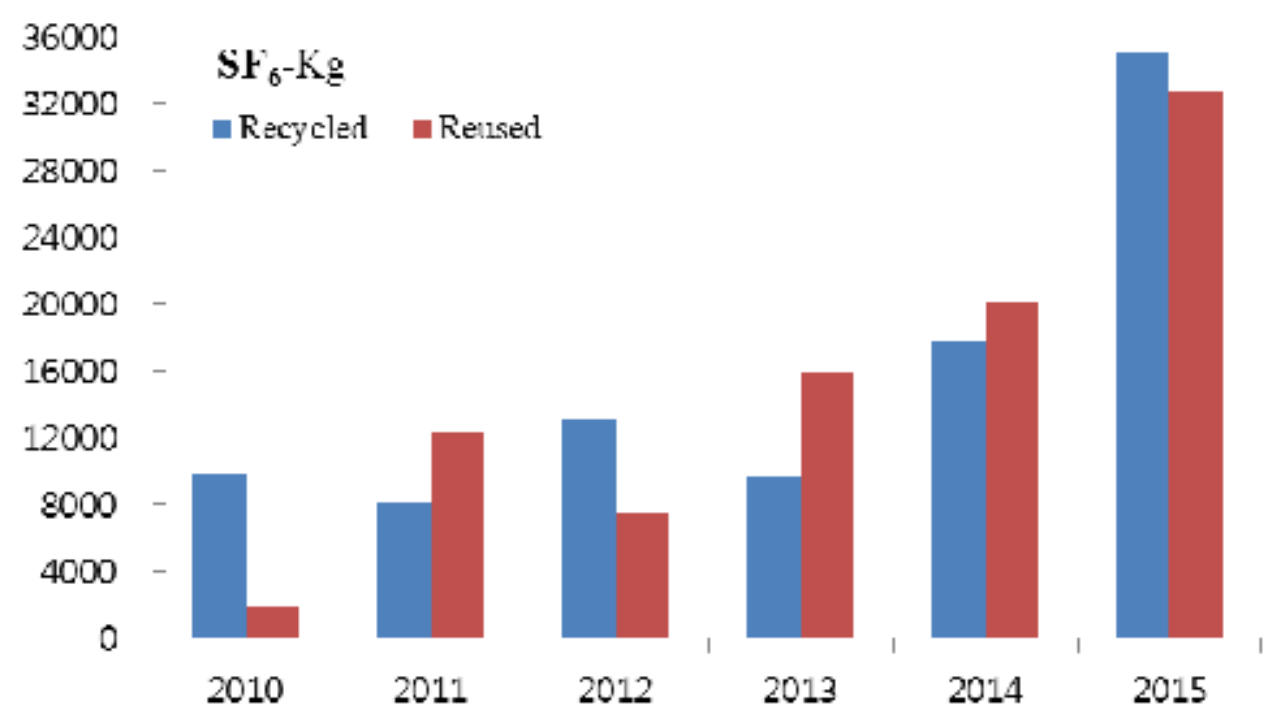

Figure. 3 Recycling and reuse of $\mathrm{SF}_{6}$ gas in Guangdong Power Grid Corporation

\section{Conclusion}

The quality standard of $\mathrm{SF}_{6}$ gas is very strict and a stricter standard is to be promulgated officially, so it is a difficult to treat large amount of $\mathrm{SF}_{6}$ gas efficiently. The $\mathrm{SF}_{6}$ Purified in Multi-channel (SFPM) technology that consist of five purify process can remove impurities, subfluoride and toxicity in disused SF6 gas, and regenerate disused SF6 gas to meet the requirements in reuse. A new kind of $\mathrm{SF}_{6}$ purification equipment was developed, a basement for Recycling and reuse of $\mathrm{SF}_{6}$ gas in Guangdong Power Grid Corporation and a great achievement was created.

The successful experience of Guangdong Power Grid Corporation shows that recycling and reuse of $\mathrm{SF}_{6}$ is technically sound, economically viable, beneficial to society and the environment, and thus important for building a green power grid and a green society.

\section{References}

[1] M. Maiss, C.A.M. Brenninkmeijer, Atmospheric SF6: trends, sources and prospects, Environ. Sci. Technol. 32 (1998), 3077-3086

[2] V. Mohindra, H. Chase, H.H. Sawin, M.T. Mocella, Abatement of perfluorocompounds (PFCS) in a microwave tubular reactor using $\mathrm{O}_{2}$ as an additive gas, IEEE Trans. Plasma Sci. 10 (1997), 399-407

[3] Wang $\mathrm{Yu}$, Differences of $\mathrm{SF}_{6}$ byproducts in simulated electric equipment of overheating faults in different condition, 1st International Conference on Energy and Environmental Protection, $\operatorname{ICEEP}(2012)$

[4] Wang Yu, Li Li, Tang Longhua, et al. Calculation on Electric Field Distribution of Typical 500 kV Current Transformer Based on ANSYS Analysis, Guangdong Electric Power(2014), 73-78

[5] M. Freemantle, Membranes for gas separation, Chem. Eng. News. 83(2005), 49-57

[6] Soonjae Lee, JongSukLee, Minwoo Lee, et al. Separation of sulfur hexafluoride ( $\mathrm{SF}_{6}$ ) from ternary gas mixtures using commercial polysulfone (PSf) hollow fiber membranes, Journal of 
Membrane Science. 452(2014), 311-318

[7] Yu-Chun Chiang, Po-Yun Wu. Adsorption equilibrium of sulfur hexafluoride on multi-walled carbon nanotubes, Journal of Hazardous Materials. 178(2010), 729-738 